\section{Selective attention and individual preferences in judgmental responses to multifeature patterns*}

\author{
CYNTHIA M. MAVRIDES \\ Laurentian University, Sudbury, Ont., Canada
}

Three experiments were conducted to show that the physical features of star-shaped patterns were preferred and used consistently as a basis of judgment across all pairs of patterns and throughout various tasks. Superior prediction of responding was achieved when it was hypothesized that within any pattern pair, the feature having the relatively larger difference in level would be emphasized in responding. In other words, both consistent preferences and selective attention to relative discriminability of features effected judgments for pattern pairs.

Responses to visual patterns are often assumed to be of a multidimensional nature, and the rationale for this assumption, as well as several concrete applications arising from it, can be found throughout the current psychophysical literature (Brown \& Andrew, 1968; Brown \& Owen, 1967; Stenson, 1968; Fenker \& Brown, 1969; Mavrides \& Brown, 1969, 1970). In most approaches it is assumed that psychological dimensions (generated and utilized by the 0 ) bear some consistent and predictable relationship to physical features of the pattern which can be measured objectively (Mavrides \& Brown, 1969, 1970).

Given that a pattern is composed of several features, research in selective attention has conclusively demonstrated that Os are not always consistent in the utilization of one single feature, or a fixed set of features (Egeth, 1967). Experiments have further demonstrated the importance of individual preferences and variability within features in the prediction of changes in selective attention (Imai \& Garner, 1965; Mavrides, 1970). It has been suggested previously (Mavrides, 1970) that selective attention might be incorporated into linear predictive models with the creation of an additional variable (dmax), which is based on the assumption that relative differences in feature levels for pairs of patterns would affect any responses based on pattern similarity. The predictive value of this assumption was demonstrated for two-feature patterns in an experiment where the effects of individual preferences in selection were minimized. The following experiments were designed to evaluate the effectiveness of the dmax variable with multifeature patterns where individual preference was an additional determinant of responding and to discover if a strategy of feature

*This research was supported by Operating Grant AP-A747 from the National Research Council of Canada.

selection (dependant on relative differences in feature levels within pattern pairs) generalized across Os and across tasks with different required responses.

\section{PATTERNS}

Patterns consisted of symmetrical star-shaped outlines (black on a white background), with points of equal angles at equal distances along the circumference of the smallest enclosing circle. The following feature measures could be calculated for each pattern: (1) The radius of the smallest enclosing circle, (2) the number of points, (3) the ratio of the radius of the largest enclosed circle to the radius of the smallest enclosing circle (indicating the depth of the stars' points), (4) the exterior interpoint angle, and (5) the interior point angle.

\section{EXPERIMENT 1}

Os for the experiment were seven undergraduate university students. Twelve patterns were constructed for the experiment, having insignificant correlations between all the five feature measures. The number of points ranged from 2 to 18 , the interpoint and point angles were all over $20 \mathrm{deg}$, the radius of the enclosing circle fell between 20 and $35 \mathrm{~cm}$, and the ratio for the enclosed circle was between .2 and .6 .

Os were presented with all possible paired comparisons, excluding those involving the same pattern twice $(\mathrm{N}=66)$. Each of the Os was tested individually and was allowed a maximum of $5 \mathrm{sec}$ to make

${ }^{*} p<01$ Table 1

Intercorrelations of the Five Difference Measures, dmax(5), and Mean Difference Judgments for Experiment 1

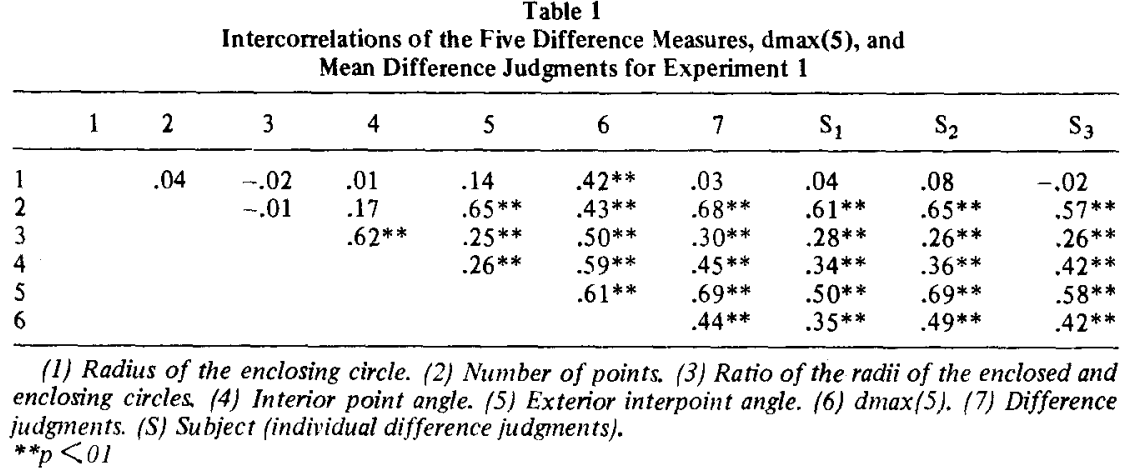

(1) Radius of the enclosing circle. (2) Number of points. (3) Ratio of the radii of the enclosed and enclosing circles, (4) Interior point angle. (5) Exterior interpoint angle. (6) dmax (5). (7) Difference judgments. (S) Subject (individual difference judgments).

an oral difference judgment with respect to each pair of patterns on a 7-point scale (1 was "very little difference" and 7 was "very much difference"). Pairs of patterns were presented on an $81 / 2 \times 11$ in. sheet of white paper, and Os were instructed to look at all pairs once before making any judgments. Pairs were presented randomly to each $\mathrm{O}$.

Since it was assumed that prediction of responses would involve measures relating the patterns in each paired comparison (rather than direct feature measures of any single patterns), an absolute difference vector was computed on the basis of all five feature measures for each of the 66 pairs. The sign of the differences was not considered important, since the order of subtraction was arbitrary and the position during presentation would not affect the magnitude of the difference. A sixth variable [dmax (5)] was added to each vector. Differences for each measure were standardized to the unit normal $(Z)$ across the 66 pairs, and dmax (5) was equal to the maximum $Z$ score in each vector. The dmax measure was designed to reflect the variability in feature level for the maximum discriminating dimension, since it was the aim of the experiment to test whether or not difference judgments would be related to a measure of this type. Data and information provided by Os (discussed below) suggested the importance of a dmax measure based on only two of the total five measures; the same method was used to generate dmax (2).

\section{Results}

The dependent variable for each pair was the mean response of the seven Os. Table 1 presents intercorrelations of the five difference measures and their correlations with judged difference. All difference measures (except for the radius of the smallest enclosing circle) correlated significantly with judgments, as did dmax (5).

When questioned after the experiment, Os claimed to have based their judgments on the number of points and the "sharpness" of points, while ignoring differences in circular extent and size. Data 
Table 2

Intercorrelations Between the Two Preferred Difference Measures, dmax(2), and Difference Judgments for Experiments 1, 2, and 3

\begin{tabular}{|c|c|c|c|c|c|c|c|c|c|c|c|c|c|}
\hline \multicolumn{4}{|c|}{ Experiment 1} & & \multicolumn{4}{|c|}{ Experiment 2} & & \multicolumn{4}{|c|}{ Experiment 3} \\
\hline 1 & 2 & 3 & 4 & & 1 & 2 & 3 & 4 & & 1 & 2 & 3 & 4 \\
\hline $\begin{array}{l}1 \\
2 \\
3\end{array}$ & $.65^{* *}$ & $\begin{array}{l}.88 * * \\
.87 * *\end{array}$ & $\begin{array}{l}.68 * * \\
.69 * * \\
.76 * *\end{array}$ & $\begin{array}{l}1 \\
2 \\
3\end{array}$ & & .38 & $\begin{array}{l}.79 * * \\
.70 * *\end{array}$ & $\begin{array}{l}.76^{* *} \\
.67 * * \\
.77^{* *}\end{array}$ & $\begin{array}{l}1 \\
2 \\
3\end{array}$ & & .38 & $\begin{array}{l}.79 * * \\
.70^{* *}\end{array}$ & $\begin{array}{l}.85^{* *} \\
.72^{* *} \\
.85^{* *}\end{array}$ \\
\hline
\end{tabular}

(1) Number of points. (2) Exterior interpoint angle. (3) dmax (2). (4) Difference judgments. $* * p<.01$

support this contention to some extent, since correlations with judgments are highest for two of the measures (the number of points and the exterior interpoint angle), suggesting a preference for their use. Since dmax (5) assumed that feature selection for each pattern pair was based on differences in level for all five features, an attempt was made to predict judgments from a dmax measure based on the two preferred features only [dmax (2)]. The correlation of $\operatorname{dmax}(2)$ with judgments was quite high (Table 2), but its correlation with each of the difference measures was also (unavoidably) high. (When the difference vector included scores of large magnitude, dmax would also be large, and the same would hold true for a difference vector having scores of small magnitude.) Partial correlations, however, indicated that much of the relationship between dmax (2) and the number of points was independent of the difference judgments (a partial correlation of .75 was obtained). The same was true of the relationship between $\operatorname{dmax}(2)$ and the exterior angle (partial $r$ was .74).

Individual Os yielded very similar responses and followed the correlation pattern of the mean difference judgments closely. Correlations with judgments for three randomly selected Os, whose data were analyzed independently, are included in Table 1 .

\section{EXPERIMENT 2}

It is noticeable that the correlation between the two difference measures best utilized by $O s$ in Experiment 1 is .65 (Table 1). Fifteen pattern pairs were selected (from the total of 66) specifically to reduce this correlation. Six Os performed a task similar to that of Experiment 1 for the 15 pairs, using a 5-point difference scale for their judgments.

\section{Results}

Correlations of judgments with three of the features (the two radial measures and the interior point angle) were insignificant. Intercorrelations of the two preferred measures, dmax (2), and the difference judgments are reported in Table 2, where it can be seen that all three variables [the two features and $d \max (2)]$ correlate significantly with judgments. In this case, the partial correlation of dmax (2) with the number of points (independent of judgments) was .48, while that of dmax (2) with the exterior angle (independent of judgments) was .37 , indicating again an interrelationship of measures not shared by the dependent variable. When dmax (5) was calculated, its correlation with judgments was .31 (not significant).

\section{EXPERIMENT 3}

Six Os were asked to order the 15 pattern pairs of Experiment 2 in five categories corresponding to the five scale values of the second experiment. They were instructed to include two pairs in the first category, three in the second, five in the third, three in the fourth, and two in the fifth. A total of $7 \mathrm{~min}$ was allowed for task completion.

\section{Results}

Correlations of the mean judgments with the number of points and the exterior angle, as well as dmax (2), are reported in Table 2. Correlations of the remaining insignificant, but the radius of the enclosing circle had a correlation of .44 with judgments. When difference judgments were partialed out of dmax (2) and the number of points, a partial correlation of .25 resulted, and the first order correlation of $\operatorname{dmax}(2)$ and the exterior angle (independent of judgments) was .40 .

Since the 15 pairs were used in all three experiments, it was possible to evaluate the consistency in judgments across tasks. The relationship of judged differences in Experiment 1 with those in Experiment 2 was .85 , and the correlation of judgments in Experiment 1 with those in Experiment 3 was .55 , while the correlation of judgments in Experiment 2 with those in Experiment 3 was .73.

\section{DISCUSSION}

The results of Experiments 1, 2, and 3 reflect a decided preference exhibited by Os in responding to pairs of star-shaped patterns. There is a consistent emphasis on the number of points and the exterior interpoint angle. It is notable that when three features with judgments were this preference is disregarded and prediction of selective responding includes feature-level differences for all five features, dmax appears to be an inferior predictor of judgmental responding. When the preference of $O s$ is taken into consideration, the dmax variable that considers differences in feature levels for the preferred features only is at least as efficient as the most efficient difference measure in predicting judgmental responses.

Further tentative support for the operation of changes in the focus of attention to features was found in the comments of Os who, when requested after the experiment to describe as much as possible their basis for responding, generally (in 15 out of 19 cases) described their behavior as "inconsistent."

Since results from the three experiments are congruent, even though two different judgmental scales and three different methods of administration were involved, it is safe to assume that any conclusions reached are not the result of task-bound artifacts. The fact that individual Os (in Experiment 1) yielded the same pattern of results as analyses of mean judgments across $O s$ seems to indicate that preferences (at least with respect to star-shaped patterns) are not completely an individual phenomenon but are held by $O s$ generally.

\section{REFERENCES}

BROWN, D. R., \& ANDREWS, M. H. Visual form discrimination: Multidimensional analysis. Perception \& Psychophysics, 1968, 3, 401-406.

BROWN, D. R., \& OWEN, D. H. The metrics of visual form: Methodological dyspepsia. Psychological Bulletin, 1967, 68, 243-259.

EGETH, H. Selective attention. Psychological Bulletin, 1967, 67, 41-57.

FENKER, R. M., JR., \& BROWN, D. R. Pattern perception, conceptual spaces, and dimensional limitations on information processing. Multivariate Behavioral Research, $1969,4,257-272$.

IMAI, S., \& GARNER, W. R. Discriminability and preference for attribures in free and constrained classification. Journal of Experimental Psychology, 1965, 69, 596-608.

MAVRIDES, C. M. Pattern perception: A predictive variable based on discriminability and selective attention. Psychonomic Science, 1970, 19, 205-207.

MAVRIDES, C. M., \& BROWN, D. R. Discrimination and reproduction of patterns: Feature measures and constraint redundancy as predictors. Perception \& Psychophysics, $1969,6,276-280$.

MAVRIDES, C. M., \& BROWN, D. R. Schematic concept formation: Feature measures and constraint redundancy as predictors. Perception \& Psychophysics, 1970, 7. 239-243.

STENSON, H. H. The psychophysical dimensions of similarity among random shapes. Perception \& Psychophysics, 1968, 3, 201-214. 